\title{
Endoluminal treatment of thoracic aortic lesions
}

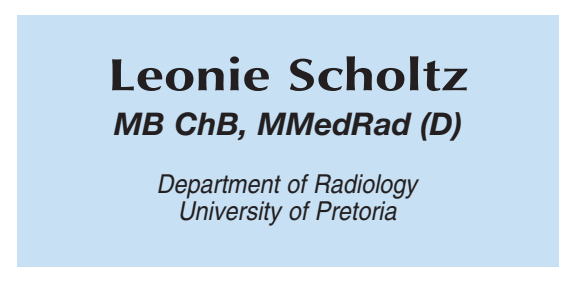

\section{Introduction}

In 1991 Juan Parodi et al. ${ }^{1}$ from Buenos Aires in Argentina placed the first endoluminal Dacron prosthetic graft in an abdominal aorta, excluding an aneurysm via retrograde cannulation of the common femoral artery. Michael Dake and his group from Stanford reported the first cases of the treatment of thoracic aneurysms. Shortly thereafter the same group started treating acute aortic dissections similarly. ${ }^{3}$ More reports of favourable results followed, ${ }^{4}$ which led to the obvious extension of the technique to the treatment of traumatic ruptures. ${ }^{5,6}$ These prosthetic stent graft devices have evolved from the initial homemade, large-profile 'beasts', to easily available, slick, readymade, low profile, user-friendly tools.

The initial worldwide, contagious enthusiasm that followed Parodi's first abdominal attempts slowly diminished, when more and more reports of late endoleaks and late ruptures surfaced. Contrary to this, however, the application of this technique in the treatment of thoracic lesions has gained ground and is slowly becoming the treatment of choice in most developed countries. The relatively uncomplicated design of these thoracic stent grafts, when compared to the bifurcated design of the aortic devices, probably accounts for the lower endoleak rate. Furthermore, the substantially higher mortality and morbidity associated with open thoracic surgery certainly swung the pendulum towards the endovascular route.

\section{Thoracic aneurysms}

The natural history of a thoracic aortic aneurysm is poor, with a rupture rate of approximately $50 \%$ when untreated. ${ }^{7-9}$ The five-year survival rate ranges from $13 \%$ to $39 \%$. The size of a thoracic aneurysm at the initial evaluation appears to be the most important predictor of rupture. ${ }^{8}$ Thoracic aneurysms seem to enlarge at a more rapid rate than abdominal aneurysms. Although surgical repair has improved, it still carries a high mortality, ranging between $10 \%$ and $16 \%$ and is associated with severe morbidity. ${ }^{10-13}$ Advanced age and emergency procedures increase mortality and morbidity. ${ }^{11}$ Complications of surgery include death, myocardial infarction, renal failure, haemorrhage, chest infections and paraplegia. Spinal cord injury is reported in $2.3 \%-6.5 \%$ of patients. The morbidity can be reduced with a less invasive procedure that spares a thoracotomy, aortic cross

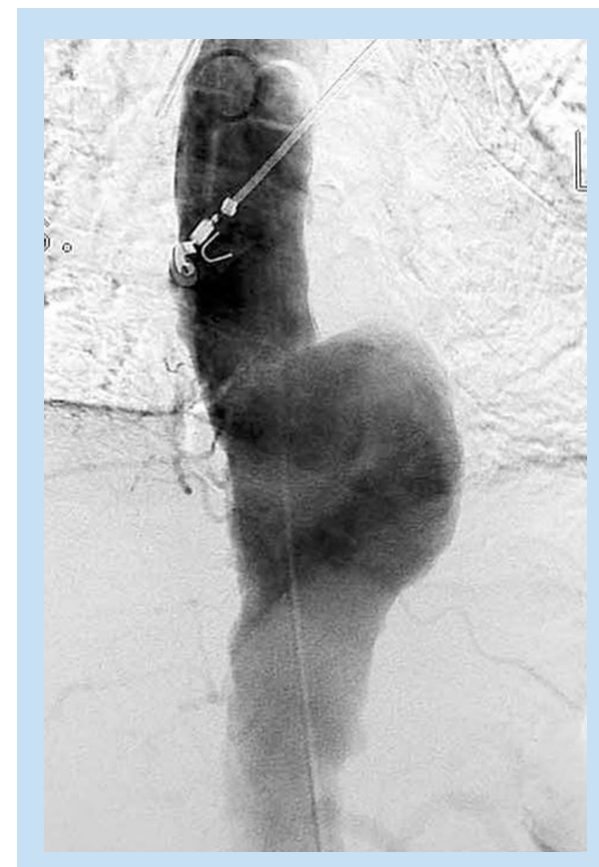

Fig. 1a. Pre-operative angiogram of a 70-year-old male patient with a degenerative aneurysm in the descending thoracic aorta.

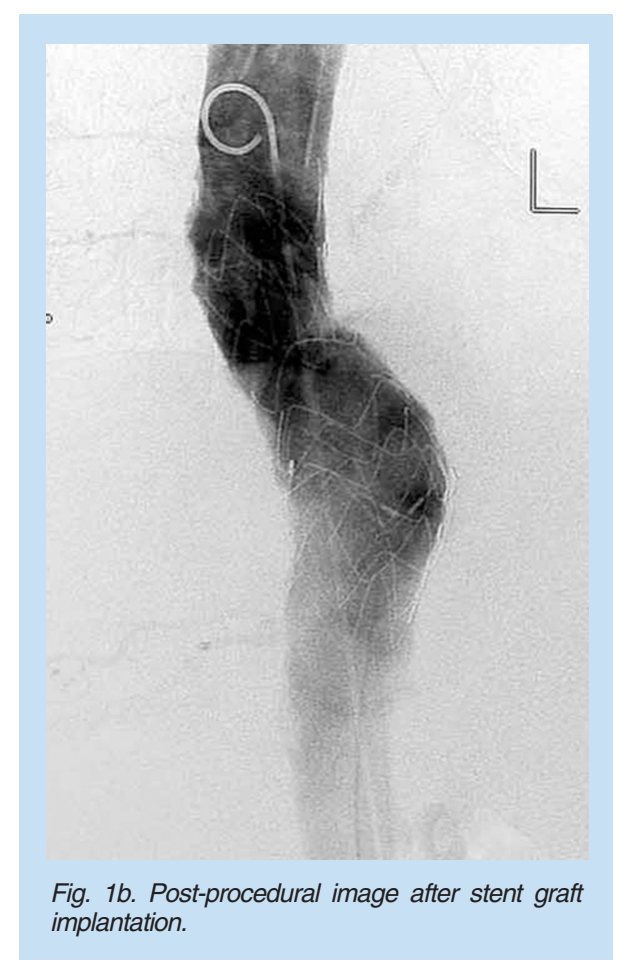

clamping and cardiopulmonary bypass. (Fig 1a, b). The mortality rate for endoluminal repair is usually $5 \%$ or less. ${ }^{14,15}$ The anaesthesia-related risks can now be minimised with 
more and more centres performing these procedures under local anaesthesia. ${ }^{16}$

\section{Thoracic aortic dissection}

Acute aortic dissection is a challenging clinical emergency first described by Morgagni more than 200 years ago. ${ }^{17}$ It is the most common acute aortic condition requiring urgent therapy. ${ }^{18}$ Classification of aortic dissection is based on anatomical location. Stanford type A dissections originate proximal to the left subclavian artery and type $\mathrm{B}$ dissections arise distal to the left subclavian artery. According to the International Registry of Acute Aortic Dissection (IRAD) the highest mortality occurred in patients with type A dissection not receiving surgery (58\%), in contrast to surgically treated patients with type A dissection (26\%). Patients with type B dissections treated medically had the lowest mortality rate $(10.7 \%)$. Mortality rate for patients with type B dissection who underwent surgery was $31.4 \%{ }^{19}$

Endoluminal stent grafts can be used to treat type B dissections and most authors report mortality rates of less than $16 \%{ }^{3,20}$ Medical therapy however remains the treatment of choice for type B dissections, unless complicated by rupture, impending rupture, end-organ ischaemia (mesenteric ischaemia, renal insufficiency or peripheral arterial insufficiency) or enlargement in the chronic phase. ${ }^{20}$ The predominant predictors for aortic enlargement in the chronic phase are the existence of a maximum aortic diameter of greater than or equal to $40 \mathrm{~mm}$ during the acute phase and a patent primary entry site in the thoracic aorta. ${ }^{21}$ (Fig 2a,b). ${ }^{21}$

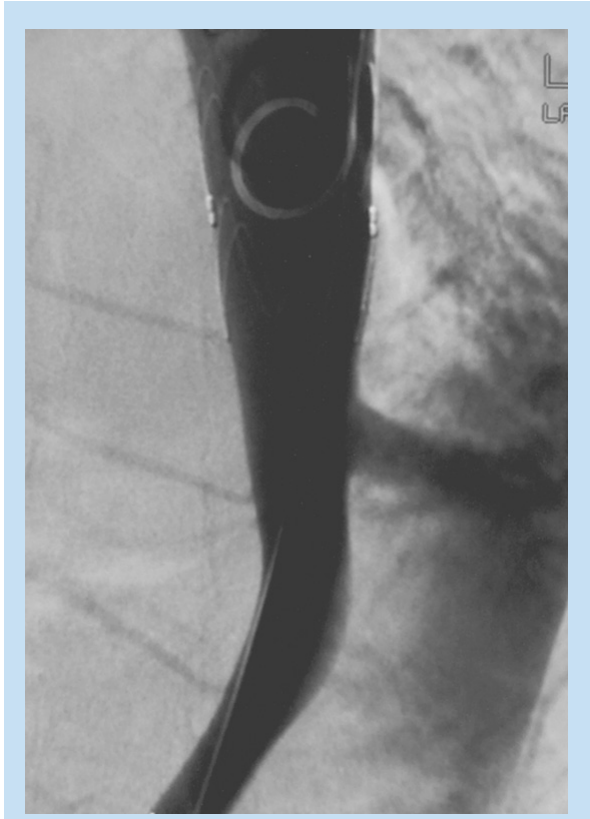

Fig. 2a. Stanford type B dissection in a 64-yearold male with the entry site in the descending aorta.

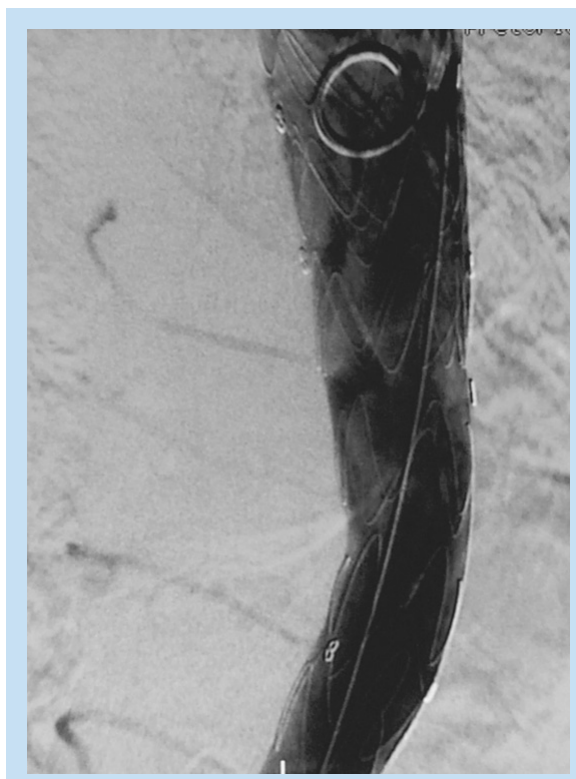

Fig. $2 b$. After coverage of the entry site with a stent graft device.

The risk of rupture is also higher in dissecting aneurysms when compared with degenerative aneurysms. ${ }^{22}$ Older age, chronic obstructive pulmonary disease and an elevated mean blood pressure increase the risk of rupture. ${ }^{23}$ Between $20 \%$ and $40 \%$ of patients who have passed their acute phase with medical hypotensive therapy will therefore require intervention during the chronic phase. Surgery still remains the treatment of choice for type A dissection.

\section{Acute traumatic rupture of the thoracic aorta}

Most patients with acute traumatic rupture of the thoracic aorta are victims of motor vehicle accidents of which $75-90 \%$ die at the scene. Approximately 15 - 20\% of the victims reach the hospital alive where blood is precariously maintained within the vascular lumen by the adventitia and mediastinal surrounding tissues. Current conventional thoracic repair is associated with a mortality rate as high as $18 \%$ and a paraplegia rate as high as $19 \% .{ }^{6}$ Where deliberate delayed repair is nowadays being advocated, endoluminal repair

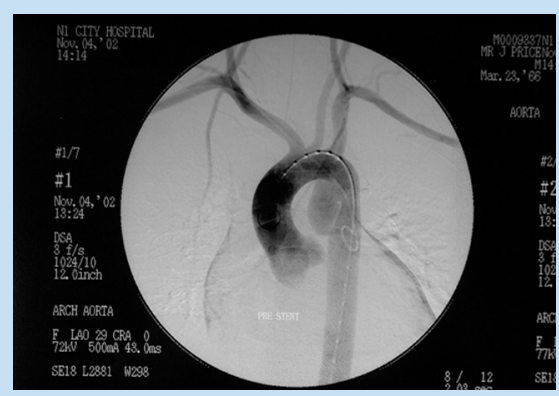

Fig. 3a. Post-traumatic pseudo-aneurysm in a 22year-old male post motor vehicle accident.

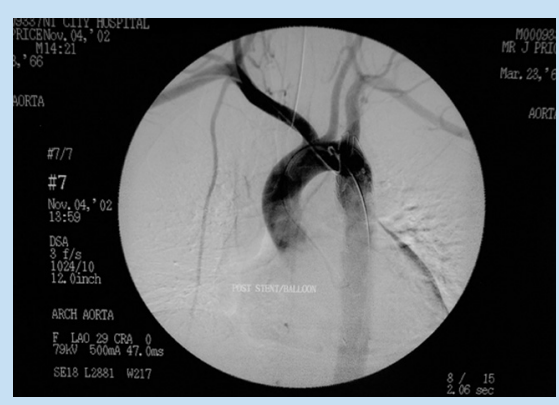

Fig. 3b. Successful occlusion of the pseudoaneurysm with an endoluminal device. 
offers the possibility of immediate repair avoiding the risk of rupture that could occur with the delayed approach (Fig. 3a,b).

\section{Diagnostic evaluation}

Patients with suspected aneurismal disease, dissection or aortic rupture require immediate diagnostic evaluation and pre-procedural work-up if endoluminal treatment is contemplated.

Contrast-enhanced multi-slice computed tomography, if available, is the most widely used diagnostic tool in the diagnosis of thoracic aortic disease. It provides information about the size, location and extent of the disease. Magnetic resonance angiography can be used although it has disadvantages in the acutely traumatised or unstable patient. Diagnostic digital subtraction angiography is reserved for cases where none of the above is available. Vital measurements and information, which have to be obtained, include: (i) the intraluminal diameter of the aorta above and below an aneurysm in perpendicular planes; (ii) locating the left subclavian artery; (iii) the length of the aorta from the origin of the left subclavian artery to where the aneurysm starts or the entry site of the dissection occurs, known as the cranial neck; (iv) the length of the aorta from below the aneurysm down to the origin of the celiac trunk, known as the distal landing zone; $(v)$ the total length of the aneurysm, or pseudo-aneurysm in the case of rupture; (vi) involvement of mesenteric branches and their origin from either the false or true lumen in cases of dissection; (vii) localisation of the entry site in dissection; and (viii) note angulation, calcification and presence of any thrombus in the cranial neck or distal landing zone.

\section{Exclusion criteria}

Exclusion criteria are; (i) cranial neck and/or distal landing zones less than $15 \mathrm{~mm}$ in length; (ii) extensive thrombus in proximal or distal landing zones; (iii) inadequate femoral or iliac access; (iv) severe angulation of more than 90 degrees in both iliac vessels; and $(v)$ severe tortuosity or angulation in the thoracic arch and descending aorta.

\section{The procedure}

Since the initial implantation of the 'first generation' homemade device at Stanford University Medical Center in 1992, major technical improvements have taken place with quite a number of commercial devices now available for deployment in the thoracic aorta. Most systems are self-expanding devices composed of a polyester graft fabric and a stent frame made from nitinol wire. Although thoracic aortic stent grafts are tube graft designs it is incorrect to conclude that they are less cumbersome to deliver and deploy.

The management team comprises a thoracic or vascular surgeon and interventional radiologist assisted by the full range of supporting cathlab and theatre personnel.

A number of anatomic caveats are relevant to the thoracic aorta and may cause procedural problems ${ }^{24}$ : (i) the thoracic aorta is frequently larger in diameter distally in the descending aorta than proximally; (ii) the descending aorta is frequently not vertically oriented and may become almost horizontal; (iii) the threedimensional features of especially the arch may cause deployment difficul- ties; and (iv) the arch can be very angulated causing difficulty in tracking such a huge device for exact positioning.

Intra-operative procedural issues include: (i) all catheters and wires need to be longer and larger; (ii) intra-operative arteriography requires larger injection rates; and (iii) one needs to be particularly meticulous in avoiding the delivery of air bubbles during the procedure.

Important procedures that should be adhered to during thoracic deployment are: (i) a pigtail catheter should be inserted preferably through the left brachial artery and placed in the arch in order to continuously check the origin of the vessels in relation to the proximal struts of the stent graft before and during deployment; (ii) we prefer general anaesthesia, although some centres perform these under local anaesthetic; (iii) open vascular access is obtained through the common femoral artery; (iv) systemic heparinisation is recommended during the procedure; $(v)$ the fluoroscopic tube should be angled to the left anterior oblique position so as to lay open the aortic neck and arch; (vi) lowering the blood pressure to a mean of $60 \mathrm{mmHg}$ during deployment and balloon inflation is recommended; (vii) a 'super stiff' guide wire is positioned across the arch of the aorta enabling stability during deployment; (viii) some centres perform pre-procedural cerebrospinal fluid drainage in an effort to minimise the risk of paraplegia; (ix) oversize the device by at least $10-15 \%$; $(x)$ only the entry site of a dissection needs to be covered; and ( $x i$ ) the left subclavian artery origin may be covered by the stent and is well tolerated in the majority of patients. 


\section{Complications of endovascular thoracic repair}

Complications of stent-graft repair include: (i) death, (ii) stroke; (iii) paraplegia; (iv) myocardial infarction; $(v)$ pulmonary insufficiency; ( $v i)$ mesenteric ischemia; (vii) renal failure; (viii) wound infection; and (ix) bleeding.

In the traditional open repair paraplegia rates of up to $21 \%$ are quoted in the literature, even with the highest level of care including intraoperative cerebrospinal fluid drainage, hypothermia, distal aortic perfusion with distal bypass and reattachment of the critical intercostals arteries. $^{24}$ Most endovascular series report rates of less than $3 \%{ }^{3,4,24}$ Preventative measures include intraoperative spinal fluid drainage as well as some centres experimenting with retrievable devices whilst monitoring evoked spinal cord potential. ${ }^{25}$

Endoleak is defined as continued flow within the aneurismal sac after deployment of a stent-graft. Type 1 endoleaks are defined as leaks at the proximal or distal attachment sites and these should be treated immediately. Type 2 endoleaks occur in the setting of patent lumbar arteries or intercostals arteries and are believed to resolve spontaneously. Late endoleaks are less common with thoracic aortic aneurysm (TAA) repair in comparison with abdominal aortic aneurysim (AAA) repair.

\section{Follow-up recommendations}

Endovascular stent-graft placement has special procedure-specific complications including stent migration, kinking and leakage that require regular surveillance. Currently, multislice CT, the imaging gold standard following endovascular repair, is performed prior to discharge and at 3, 6, 12 , and 24 months after intervention, thus causing significant radiation to the patient as well as adding cost. MRA has the potential to become an alternative imaging modality in the thoracic aorta, whereby the risk of radiation can be reduced. Susceptibility artifacts associated with stents still pose problems as well as certain potential safety issues including magnetic field interactions that may move or dislodge the stent and heating of the endoprosthesis by radio frequency (RF) power deposition. The 3D Turbo FLASH sequence provides the least susceptibility artifact due to a short echo time and a small effective slice thickness.

\section{Conclusion}

Endoluminal repair of thoracic aortic aneurysms, type B dissections and acute rupture is becoming the treatment of choice worldwide. More and more devices are now commercially available with designs continually being refined. Lower profiles, flexibility and ease of deployment make them user-friendly, with low complication rates and high primary technical success rates. Morbidity and mortality rates are substantially lower when compared with open surgical repair and endoleaks rare when compared with abdominal procedures. A definite disadvantage currently is the cost of the devices, which amounts up to anything between R50 000 and R100 000 depending on the amount of main devices or extensions used. If however, lengthy ICU-stay and the overall cost implications in treating postoperative surgical complications are taken into account, the cost probably compares favourably with open repair.

\section{References}

1. Parodi JC, Palmaz JC, Barone H D. Transfemoral intra-luminal graft implantation for abdominal aortic aneurysm. Ann Vasc Surg 1991; 5: 491499.

2. Dake MD, Miller DC, Semba CP, et al. Transluminal placement of endovascular stent grafts for the treatment of descending thoracic aortic aneurysms. NEngl J Med 1994; 331: 17291734.

3. Dake MD, Kato N, Mitchell RS, et al. Endovascular stent-graft placement for the treatment of acute aortic dissections. $N$ Engl J Med 1999; 340: 1524 - 1531.

4. Nienaber CA, Fattori R, Lund G, et al. Non surgical reconstruction of thoracic aortic dissection by stent graft placement. NEngl J Med 1999; 340: 1539 - 1545 .

5. Lachat M, Phammatter T, Witzke H, et al. Acute traumatic aortic rupture: early stent graft repair. Eur J Cardiothorac Surg 2002; 21: 959 - 963.

6. Thompson CS, Rodriguez JA, Ramaiah VG, et al. Acute traumatic rupture of the thoracic aorta treated with endo-luminal stent grafts. J Trauma 2002; 52: 1173 - 1177.

7. Pressler V, McNamara JJ. Thoracic aortic aneurysm : Natural history and treatment. Thorac Cardiovasc Surg 1980; 79: 489 - 498.

8. Perko M J, Norgaard M, Herzog M, Olsen PS, Schröder TV, Pettersson G. Unoperated aortic aneurysms: a survey of 170 patients. Ann Thorac Surg 1995; 59: 1204 - 1209.

9. Pressler V, McNamara JJ. Aneurysms of the thoracic aorta. J Thorac Cardiovasc Surg 1985; 89: 50-54.

10. De Bakey ME, McCollum CH, Graham JM. Surgical treatment of aneurysms of the descending thoracic aorta. J Cardiovasc Surg 1978; 19: $571-576$.

11. Livesay JJ, Cooley EA, Ventemiligia RA, et al. Surgical experience in descending thoracic aneurysmectomy with and without adjuncts to avoid ischeamia. Ann Thoracic Surg 1985; 39: 3746.

12. Borst H G, Jurmann M, Bühner B, Laas J. Risk of replacement of descending aorta with a standardized left bypass technique. J Thorac Cardiovasc Surg 1994; 107: 126 - 133.

13. Von Segesser LK, Killer I, Jenny R, Lutz U, Turina MI. Improved distal circulatory support for repair of descending thoracic aortic aneurysms. Ann Thorac Surg 1993 ; 56: 1373 - 1380.

14. Mitchell RS, Dake MD, Semba CP, et al. Endovascular stent-graft repair of thoracic aortic aneurysms. J Thorac Cardiovasc Surg 1996; 111: 1054 - 1062.

15. Ehrlich M, Grabenwöger M, Grimm M, et al. Endo-vascular stent-graft repair for aneurysms of the descending thoracic aorta. Ann Thorac Surg 1998; 66: 19-24.

16. Lachat M, Pfammatter T, Turina M. Transfemoral endografting of thoracic aortic aneurysms under local anaestheasia : a simple, safe and fast track procedure. Vasa 1999; 28: 204 $-206$. 
17. Acierno LJ. The History of Cardiology. New York, Parthenon Publishing Group, 1994.

18. Pretre R, Von Segesser LK. Aortic dissection. Lancet 1997; 349: 1461-1464.

19. Hagan PG, Nienaber CA, Isselbacher EM, et al. The international registry of acute aortic dissection (IRAD). New insides into an old disease. JAMA 2000; 283: 897 - 903.

20. Shimono T, Kato N, Yasuda F, et al. Transluminal stent-graft placements for the treatment of acute onset and chronic aortic dissections. Circulation 2002; 106: 241-247.

21. Kato M, Bai H, Sato K, et al. Determining surgical indications for acute type $\mathrm{B}$ dissection based on enlargement of aortic diameter during the chronic phase. Circulation 1995; 92: Suppl II: 107 - 112.

22. Griepp RB, Ergin MA, Galla JD, et al. Natural history of descending thoracic and thoraco-abdominal aneurysms. Ann Thorac Surg 1999; 67: 1927 - 1930.

23. Juvonen T, Ergin MA, Galla JD, et al. Risk factors for rupture of chronic type B dissections. J Thorac Cardiovasc Surg 1999; 117: 777 - 786.

24. Milner R, Bavaria JE, Baum RA, et al. Thoracic aortic stent grafts. Semin Roentgenol 2001; 4: 340-350.

25. Ishimaru S, et al. Preliminary report on prediction of spinal cord ischemia in endovascular repair of thoracic aortic aneurysm by retrievable stent graft. $J$ Thorac Cardovasc Surg 1998; 115: 811-818.

\section{Further reading}

1. Ince H, Rehders TC, Nienaber CA. Thoracic aneurysms and type B dissections should be treated by stent graft. In: Greenhalgh RM, ed. The Evidence For Vascular or Endovascular Reconstructions. WB Saunders, 2002: 119-125.

2. Kouchoukos NT, Dougenis D. Surgery of the thoracic aorta. N Engl J Med 1997; 336: 1876 - 1888.

3. Anagnostopoulos CE, Prabhakar MJ, Kittle CF. Aortic dissections and dissecting aneurysms. Am J Cardiol 1972; 30: 263 - 273.

4. Vann JI, Sarres GE, Mitchell RS, et al. Treatment of patients with aortic dissection presenting with peripheral vascular complications. Ann Surg 1990; 212: $705-713$

5. Genoni M, Paul M, Tavakoli R, et al. Predictors of complications in acute type B dissection. Eur J Cardiothorac Surg 2002; 22: 59-63.

6. Sueyoshi E, Imana T, Sakamoto I, et al. Analysis of predictive factors for progression of type B aortic intra-mural heamatoma with computed tomography. J Vasc Surg 2002; 35: 1179 - 1185.

7. Marui A, Mochizuki T, Mitsui N, et al. Towards the best treatment for uncomplicated patients with type B acute aortic dissection. Circulation 1999; 100: Suppl II, 275 - 280.

8. Lansman SL, Hagl C, Fink D, et al. Acute type B aortic dissection: surgical therapy. Ann Thorac Surg 2002; 74: S1833 - 1835.

9. Sasaki S, Yasuda K, Kunihara T, et al. Surgical results of standard type B aortic dissection. Comparison between partial and subtotal replacement of the dissected aorta. J Cardiovasc Surg 2000: 41: 227 -232.

10. Umana JP, Miller DC, Mitchell RS. What is the best treatments for patients with acute type B aortic dissections. Medical, surgical or endovascular stent grafting? Ann Thorac Surg 2002; 74: S1840 - 1843.

11. Lonn L, Delle M, Lepore V, et al. Endograft therapy of the thoracic aorta in aortic dissections. Cardiovasc Intervent Radiol 2002; 25: suppl II S158.

12. Lee DY, Chou DH, Shim WH, et al. Elective endovascular treatment of aortic dissections with stent grafts. Cardiovasc Intervent Radiol 2002; 25: suppl II, S158. 